\title{
ETHICO-AESTHETIC KNOW-HOW: THE ETHICAL DEPTHS OF PROCESSUAL ARCHITECTURE
}

\author{
PIA EDNIE-BROWN \\ School of Architecture and Design \\ RMIT University \\ PO Box 2476V, Melbourne, VIC 3001, Australia \\ pia@rmit.edu.au
}

\begin{abstract}
This paper argues for an ethics embedded in the largely digitally oriented field of research I refer to as 'processual architecture', in terms of Francisco Varela's notion of ethical expertise. Processual architecture has been a prominent field of architectural research whose depth of value and substance has eluded many. After the last 15 years or so of digital experimentation, it is clear that digital technology in itself is not the primary issue, but simply part of an equation. The ethical implications of this equation, I argue, can be found through the affinity between Varela's ethical expertise and an idea of the art of emergence. Emergence, a construct that describes a contemporary version of the laws of nature, has been used with increasing prominence in architecture in recent years.
\end{abstract}

Keywords: digital, ethics, aesthetics, emergence, architecture

\section{Introduction}

"To tend the stretch of expression, to foster and inflect it rather than trying to own it, is to enter the stream, contributing to its probings: this is co-creative, an aesthetic endeavour. It is also an ethical endeavour, since it is to ally oneself with change: for an ethics of emergence." (Massumi xxii)

The field of architectural research and practice significantly employing digital technologies in explorative, experimental ways is commonly discussed under the banner 'digital architecture'. I prefer, however, to use the term 'processual architecture'. This naming dislodges the centrality of digital technology as that which defines the field, without removing its significant role. It also acknowledges its association with process philosophy (see Rescher; Gare) and processual media theory (Rossiter) and the related tendency to privilege or highlight things 'in-process'. Through early proponents such as John Frazer and, more contagiously, Greg Lynn and others, the formation of the field was characterised by animated diagrams and kinetic objects, moving and gyrating in differentiating fields of data. All the work that defines the field of processual architecture foregrounds - in various ways and degrees - processes of formation as constituting key properties or defining conditions of the product. Built outcomes become emphatically responsive, interactive and/or experientially challenging, while design processes become products in themselves (known as 'designing the design'). As a somewhat aesthetically 
re-inflected extension of the emphases on process and responsiveness in the 1960s, this field of work operates to further unsettle the clarity of distinction between process and product.

While an appreciation of processual architecture involves the new opportunities being explored through digital technology, there is clearly much more at stake than technology and technique. While this might seem obvious in itself, an articulation of a broader and richer project implicit to the field is hard to find. The depth of its value and substance has been far from obvious to many. Its perceived superficiality often arises from the fact that most of the work demonstrates a focus limited to a sophisticated aesthetics of form - sculptural form and/or the sculpted form of a process - at the expense of the socio-cultural or environmental concerns explored in other areas of architectural practice. Some commentators have gone so far as to suggest that the work is unethical (Ostwald; Perez-Gomez). I will argue a different case here.

To suggest that a field of blobs and arty interactives contain ethical potential might at first feel a little jarring. But only so long as processual architecture continues to be considered superficially, because its sophisticated surfaces of appearance can involve a behavioural composition of considerable depth.

The case I make begins with the model of emergence and its resonance with both processual architecture and the broader socio-cultural situation of our present era. While the topic of emergence in this field of architectural discourse goes back to its shimmeringly youthful stirrings in the early 90's (Kwinter) and has, I would argue, implicitly moulded the general experimental agenda since that period, it has been referred to with increasing intensity over the past 3-5 years. This is particularly evident in a cluster of younger or more recent architectural practices, such as biothing, kokkugia, Ruy Klein, Arandah/Lasch and the Emergence and Design Group who are explicitly claiming processes of emergence as central to the concerns of their practices.

\section{Emergence as a model of composition}

The term 'emergence' refers to a model of the fundamental operations of the world and is seen to be "a ubiquitous feature of the world around us" (Holland 2). As such, emergence becomes the name for a contemporary understanding of the laws of nature. As a discursive construct it seeks to explain, often through mathematical frameworks, the way that complex, sophisticated forms of organization come into being through simple, local behaviours and rules, in the absence of any apparent, centralized or dominant control mechanism. The classic examples are ants and cellular automata: where multitudes of individual 'agents' act via simple behavioural rules that together self-organise into patterns or forms of intelligence. A powerfully significant feature of emergence is that it is no less applicable to economic systems, games and urban planning than it is to living and natural systems. With emergence, culture and nature are artfully swept together.

Significantly here, emergence provides a model that describes the nature and organisation of contemporary socio-cultural operations particularly well, and vice versa. It becomes an academic construct for approaching the atmosphere of our present era and defining, to some extent, what it means to be 'contemporary'.

Let's think about the field of our cultural present. The broad socio-economic atmosphere in which we are situated seems to increasingly display emergent behaviour: our systems operating as highly responsive, decentralised, self-perpetuating networks demonstrating life-like activity to which no simple cause and effect relations can be attributed. Think of the stock market, reality $\mathrm{TV}$, the intertwinement of media, politics and the multitude, terrorism, climate change, mobile phone swarming. These very contemporary situations are examples of phenomena engaged in perpetual on-going emergence. Emergent phenomena are, in a sense, the elusive products of a virtuosic event or culture, but as a 'product' it is inseparable from the multiplicity that creates it and pervasive in that it modulates that very multiplicity. The moment it emerges out of a field it curls back in. In a manner that has been described as "viciously circular" (Bedau 16), there is a feedback of affects between that which emerged and that from which it emerges.

From its earliest conjectures, the issue of emergence has been tied up with the battles between theories of evolution and creationism; the world as machine and the existence of God. It is a construct that seeks to explain how novelty arises, whether that be new species of life, innovative theories or technical objects. As such, emergence intrinsically concerns the process of how things are created or generated and the constitution of that process. In other words, emergence is an issue of composition: the process and outcome of combining things to form a whole. 
As soon as that connection is made, the notion of composition as a formal arrangement of parts is given a processual or performative spin, because emergence models processes of interaction or the dynamics of unfolding relations. Composition becomes a performance and pertains to forms only in terms of the dynamic relations in which they are engaged. Rather than composition pertaining to, say, the formal arrangement of a facade, it pertains to the process through which that façade comes into being, the sense of that process in the designed product and the ways in which this performs after being built. While not precluding the possibility of remaining computationally describable at some level, this dynamic compositional glue becomes like the feeling of pleasure and the mannerisms that animate the face into a smile rather than the formal arrangement of a smiling face. And like the dancer, the designer can't perform well without an attention to the transmission of affect between bodies and influences and a practiced grasp of a style of movement. While this is acutely important in the case of the designer, it is also relevant to the subsequent engagements of others with the design products.

\section{Ethical Expertise and the Art of Emergence}

In his book, Ethical Know-How. Action, Wisdom and Cognition, Francisco Varela proposes a model of ethical expertise or know-how. Most western writers on ethics, he claims, tend to focus on reasoning as the central issue wherein ethics becomes an issue of deliberation (23). Ethical expertise, on the other hand, does not centre itself on rational judgements of reasoning or on how this may be applied as ethically instrumental. Rather, it is based on the inextricability of the specific tissue of circumstances or situatedness. With some affinity with Foucauldian and Spinozist approaches to ethics, as well as Felix Guattari's notion of the ethico-aesthetic, his notion of ethical expertise dwells in a "skillful approach to living ... based on a pragmatics of transformation that demands nothing less than a moment to moment awareness of the virtual nature of our selves" (75). To act ethically, one must behave with sensitivity to the particularities of the situation where there is not a reliance on a set of rules:

"To gather a situation under a rule a person must describe the situation in terms of categories we may call cognitive. Instead, if we try and see correspondences and affinities, the situation at hand becomes much more textured" (28).

Along these lines, Varela has suggested that "intelligence should guide our actions, but in harmony with the texture of the situation at hand...truly ethical behaviour takes the middle way between spontaneity and rational calculation" (31-32).

Described as such, ethical expertise has a close affinity with 'the art of emergence'. Resonating with comments from a number of complexity theorists (Holland; Taylor), Steven Johnson writes that:

"We are only just now developing such a language to describe the art of emergence. But here's a start: great designers ... have a feel for the middle ground between free will and the nursing home, for the thin line between too much order and too little. They have a feel for the edges" (189).

It would seem that the art of emergence involves what Varela calls ethical expertise, that together can be usefully referred to as ethico-aesthetic expertise. I should emphasise here that ethics is not about the 'good' and the 'bad', redemption or claims for redemptive powers. Its about a 'measured' practice of engaging with the world, of how we behave, of what we acknowledge is at stake. Ethico-aesthetic expertise is about the amplification of potential which doesn't necessarily lead to the 'good' because it magnifies risk. Rather than being framed around the virtuous, ethico-aesthetic expertise is about the virtuouso: the skilled performer (Virno). Even if there are no easy rules or moral guidelines here, there is an important principle or navigational directive. That is: that the performance of any act strives for a balance between affecting and being affected, between active reflection and the immediacy of embodied response, between sensitive responsiveness and determined agency. This is a politics of action that neither caves in passively to collective desires or beliefs nor holds to individualism, authorship or dictatorship as the power of truth. It is both determined and respectful, pushy and playful. It involves raising both thinking and acting to their highest powers, such that they affect and fold into one another. Or, in short, it involves the embodiment of wisdom.

\section{The Ethico-aesthetic Know-How of Processual Architecture}

Processual architecture characteristically focuses on setting up conditions or generative systems through which outcomes will emerge. This often involves the inter-relational arrangement of micro-components or agents, each of which are programmed with behavioural 
rules. The performance of the designer is met with dynamic, life-like diagrams that are themselves configured in terms of behaviours and performance. The strength of the life-like nature of these diagrams (or abstractly experienced objects) means that they become like puppets that the designer guides, but with enough in-built character to take a part in leading or guiding the way. In other words, the design material is not passive but pushy, involving a dynamic between designer and the designed wherein each both affect and are affected by one another. Implicitly, composition becomes less of a set of rules or methodologies and more of an ethics of engagement or ethico-aesthetic expertise.

Ethical dimensions, that others have judged to be missing, are embedded in the way that these emphatically processual architectures foreground and bring into question the nature of our engagements in the world. While all architecture implicitly raises this question, actually (and virtually), the difference here lies in the amplified attention to our engagements that processual architecture ushers into the scene.
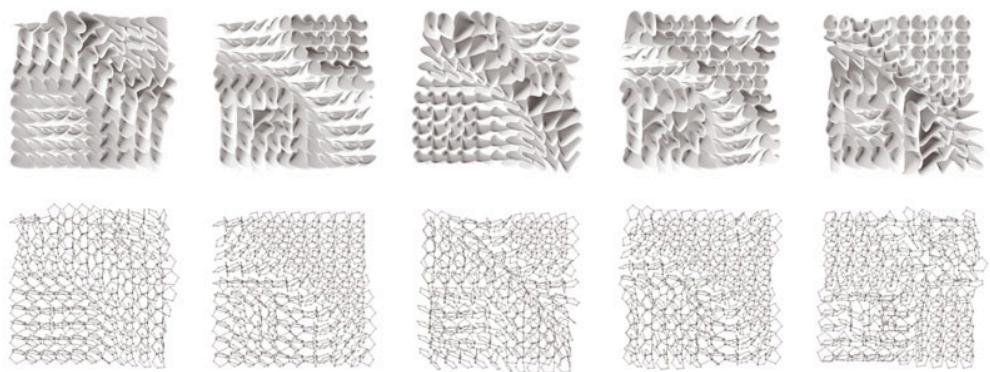

Image 1: Alisa Andrasek (biothing), The Invisibles (2003)

A specific example of the tendencies of processual architecture that I'm referring to here, can be found in the work of biothing or Alisa Andrasek. In a previously published analysis of one of her projects, The Invisibles, I argued for a new mode of composition symptomatic of processual architecture (Ednie-Brown). In that essay I discussed how the digital field system of The Invisibles is emphatically, even if invisibly, part of a broader event-ecology through which parameters that do not take the form of digital data nevertheless participate. This involves a complexity of project criteria of many different kinds, along with the tendencies of the designer, knitted together by her habits, attentions, memories, affections and so on - a cluster of potential that can be folded into what we call 'personality', described by Brian Massumi as a 'pattern of preferential headings' (205).

Personality enters into a dance with the potentials of the medium of design manipulation, accompanied by a range of other pragmatic and intangible influences. In the event of negotiating an undulating ground of criteria-meeting-potential, the designer becomes part of a depth of complex relationality such that the totality of the compositional event becomes one evolving 'thing'. Within this larger thing, the developing digital system is a material in the making.

In the cacophony of such an event there is a striving that tempers development: to create or compose this material: the morphologically dynamic system that constitutes the product. The designer leads, but not bluntly or brutally. Likening the process to the training of a pet, Andrasek talks about 'teaching it, guiding it, stirring in certain directions, but at the same time learning from IT' (Andrasek). IT's 'life' flickers forward when IT begins to develop a 'pattern of preferential headings' or an abstract 'personality'. As IT comes-into-being, the compositional event bifurcates into a clarity of differentiation between her and IT. She is no longer the only source of 'push' amidst a scattering of material because she now has an 'IT' (a system) to play with. 


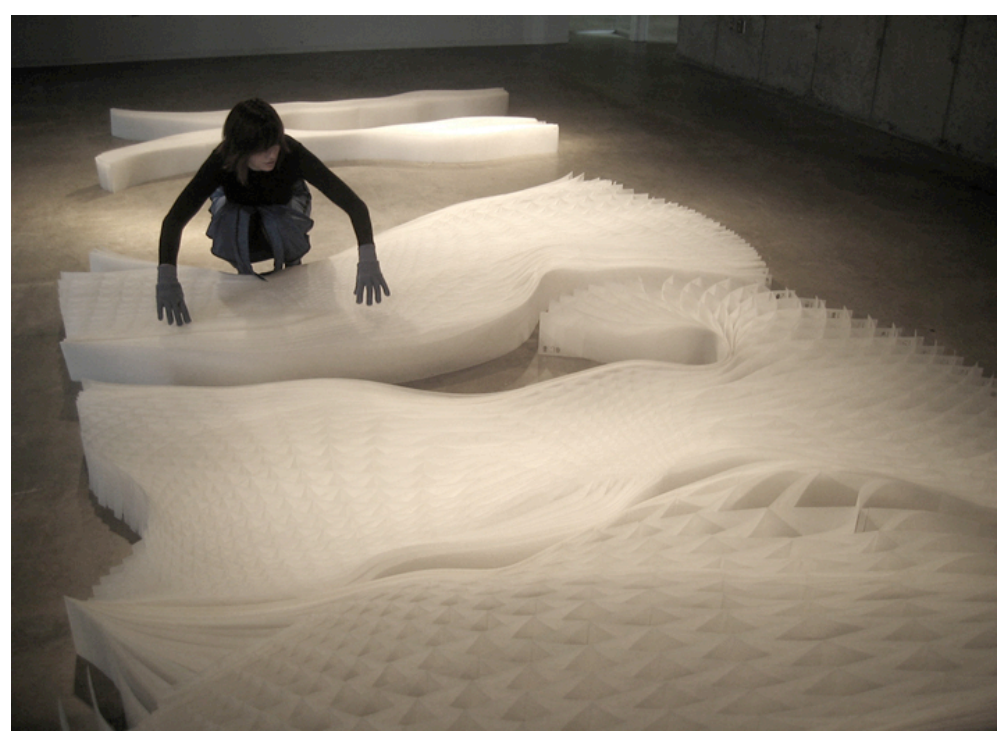

Image 2: Alisa Andrasek and her algorithmic fabric 'creature' (bifid v1.5)

It becomes, as I like to describe it, a system defined by tendencies of behaviour that give it a consistency. By consistency I mean the sort of thing we refer to when discussing the consistency of a cake mix. Rather than some idea of sameness or uniformity, consistency is the texture arising from the way in which something dynamically holds together. The strength of this consistency means that it develops enough behavioural tendency (or 'patterns of preferential headings') to have character, becoming something of a creature, a term Andrasek often uses to refers to her design systems.

These creatures are constituted by multitudes of dynamic micro-interrelations. In both an experience of and an engagement with these creatures the relations that constitute them cannot be singled out: they are never experienced in isolation, not even as some part of a whole. As a multitude engaged in an emergent process of composition, they generate patterns or textures of multiple, mostly invisible, relations: an intricate consistency. So, what we (aesthetically) experience is an all-over, over-all consistency.

The power of this qualitative envelope is such that invisible forces become undeniable. There is a forcefulness of coordinated consistency through which the dynamics of complex relationality becomes explicitly articulated. When something can no longer be denied one has to engage with its difficulties and inconvenient challenges (like climate change). Learning how to engage with a complex, wilful and difficult intricacy of relations is tantamount to opening oneself up to a depth of relationality in the tissue of any circumstance. Such an embrace, often denied in order to maintain the ideality of 'control', is crucial to the development of ethico-aesthetic expertise.
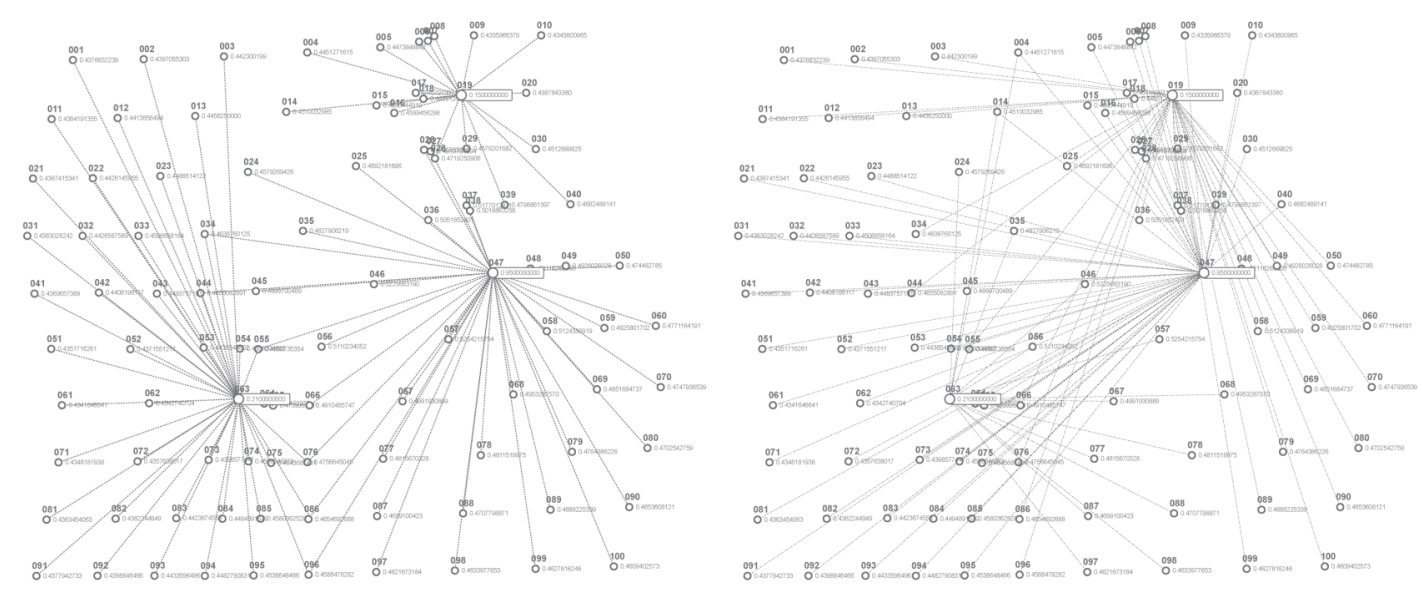

Image 3: Alisa Andrasek - diagram of algorithmic speed-distribution and cellular relationships: The Invisibles (2003) 
In part, one could say that its all about finding a 'balance' between the polar 'opposites' of all conditions (too much order and too little, formality and informality, the one and the many, etc etc). But these are less opposites than categories that define the limits of various states of relation. 'Balance' does not equate to stillness or sweet, peaceful composure, because it might tend more toward a wildly oscillating performance of relation. It is rare for situations to be free of struggles to connect, conflicts of interest/affect or obstacles to sharing/engaging. Extremes of these conditions lead to various forms of war, but milder instances are everywhere: in the dynamics of relation, relevant to political, social, personal and creative assemblages such as the design event.

Design practices that emphatically work with performative, dynamically relational systems provoke a demand for an ethico-aesthetic expertise. Pedagogically, this becomes very significant, in that it raises the issue of how to help students develop this form of expertise. This is less something that can be taught directly than ushered into the studio environment, through the manner of one's engagements with the students and the material they generate, providing a framework that is both firm enough to provide constraint and responsive enough to adapt to material and issues that arise, and guiding design developments such that a productive resonance arises between the design process, the performance of the outcome and an ethicoaesthetic expertise. This approach to teaching is difficult and risky but close to the practice that Sandford Kwinter discusses as the 'cultivation of life', where one must bear the knowledge that, as he writes, we cannot know "where such an experiment will go, and it is one certainly rife with traps and dead ends."(37)

For the sake of pedagogy and discourse, an ability to keep the risk alive while managing to recognise the dead ends when they arise, to twist traps into realisations, and to match firmness with sensitivity, might enable processual architecture to more actively (and ethically) contribute to issues well beyond its usual focus on formally articulated virtuosity - by virtue of its virtuosic capabilities.

Or it might not. But it's worth the risk.

\section{References}

Andrasek, Alisa, email correspondence with Pia Ednie-Brown, 22 December, 2004.

Bedau, Mark, 'Downward Causation and the Autonomy of Weak Emergence', Principia 6, . (2002): 5-50.

Ednie-Brown, Pia, 'All-Over, Over-All: biothing and Emergent Composition', Programming Cultures: Art and Architecture in the Age of Software, Helen Castle, Michael Silver (eds), London: Architectural Design Academy Editions, No 182, Vol 76, no 4, July/August, (2006): 72-81.

Gare, Arran, 'Process Philosophy and the Emergent Theory of Mind: Whitehead, Lloyd Morgan and Schelling', Concrescence 3, (2002): 1-12

Holland, John, Emergence. From Chaos to Order, New York: Basic Books, 1999.

Johnson, Stephen, Emergence: The Connected Lives of Ants, Brains, Cities and Software, NewYork: Schribner, 2004.

Kwinter, Sanford, 'Emergence: or the Artificial Life of Space', Anywhere, Cynthia C. Davidson (ed), New York: Rizzoli, (1992): 163-171.

Kwinter, Sanford, 'The Gay Science (What is Life?)', Life Style, Bruce Mau, Phaidon Press Limited, London, 2000.

Massumi, Brian, Parables for the Virtual. Movement, Affect, Sensation, Durham and London: Duke University Press, 2002.

Ostwald, Michael, 'Freedom of Form: Ethics and Aesthetics in Digital Architecture', The Philosophical Forum, Vol XXXV, No 2, (2004): 201-220

Perez-Gomez, Alberto, Built upon Love: Architectural Longing after Ethics and Aesthetics, Cambridge: MIT Press, 2006.

Rescher, Nicholas, Process Philosophy: A Survey of Basic Issues, University of Pittsburgh Press, 2000 
Rossiter, Ned, Processual Media Theory: Organised Networks and the Politics of Information Societies, PhD thesis, School of Communications and Multimedia, Faculty of Communications and Creative Industries, Edith Cowan University, 2004

Taylor, Mark C, The Moment of Complexity: Emerging Network Culture, Chicago, London: University of Chicago Press, 2001.

Varela, Francisco J, Ethical Know-How. Action, Wisdom, and Cognition, Stanford: California, 1999.

Virno, Paolo, A Grammar of the Multitude, James Cascaito, Isabella Bertoletti, and Andrea Casson (trans.), New York: Semiotext(e), 2004. 